\title{
ComPlexUs
}

\section{NETWORK MODELLING}

Complexus 2006:3:169-182 DOI: 10.1159/000094198

Published online: August 25, 2006

\section{Correlation Model of} Worm Propagation on Scale-Free Networks

\author{
Zoran Nikoloski ${ }^{a} \quad$ Narsingh Deo ${ }^{b} \quad$ Ludek Kucera $^{a}$ \\ ${ }^{a}$ Department of Applied Mathematics, Faculty of Physics and Mathematics,

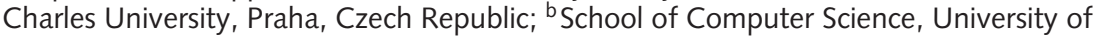 \\ Central Florida, Orlando, Fla., USA
}

\section{Key Words}

Propagation $\cdot$ Network worm $\cdot$ Scale-free graph $\cdot$ Correlation model

\begin{abstract}
The problem of network worms is worsening despite increasing efforts and expenditure on cyber-security. Worm propagation is a random process that creates a complex system of interacting agents (worm copies) over the propagation medium - a scale-free graph, representing real-world networks. Understanding the propagation of network worms on scale-free graphs is the first step towards devising effective techniques for worm quarantining. After presenting the drawbacks of existing mean-field models, we develop a pair-approximation (correlation) model of worm propagation that employs the salient network characteristics - order, size, degree distribution, and transitivity. Inclusion of the transitivity shows significant improvement over existing pair-approximation models. The validity of the model is confirmed by comparing the numeric solution of the model to results from our individual-based simulation. Our model demonstrates that the network structure has considerable impact on the propagation dynamics when the worm uses local propagation strategies.
\end{abstract}

Copyright $\odot 2006$ S. Karger AG, Basel

Fax +41613061234 E-Mail karger@karger.ch www.karger.com

KARGER (c) 2006 S. Karger AG, Basel 1424-8492/06/0033-0169 $\$ 23.50 / 0$

Accessible online at: www.karger.com/cpu
Zoran Nikoloski, Department of Applied Mathematics, Faculty of Physics and Mathematics, Charles University, Malostranske Nam. 25, CZ-118 00 Praha 1 (Czech Republic) Tel. +420 221914 233, Fax +420 257531014 , E-Mail nikoloski@kam.mff.cuni.cz

\section{Simplexus}

From email 'spam' wasting valuable network bandwidth to the theft of personal identities, cyber-security today demands increasing attention. Among the most serious and pressing problems is that of socalled digital 'worms' - programs that hijack computers and use them to send copies of themselves to other computers. In the summer of 2001, a tiny bit of code known as the Code-Red (CRv2) worm infected more than 359,000 computers in just over half a day, spreading like a real virus, but using electronic means of transmission. The threat such worms pose grows more severe each year, and there is probably no simple and final solution. We will have to fight electronic viruses in the same way we do biological ones - with our own techniques of ever-advancing sophistication.

However, it is equally clear that the development of such techniques can only benefit from a better understanding of the mechanics of worm movement. As Nikolski and colleagues note in this paper, a striking feature of the networks through which worms travel - either the physical Internet, or the networks of email contacts that link individuals together - is their 'scale free character'. The nodes in such networks do not have a well-defined 'average' number of links to other nodes; rather, the number of links possessed by a node is highly variable across the network. Although some attempts have been made to extend traditional models of epidemiology to apply to such scale free networks, researchers still do not have models that produce accurate results with anything short of large-scale simulations. In this paper, the authors make a contribution in this direction by demonstrating the accuracy of a coarse-grained model for worm spreading on scale-free networks. In particular, they show that the results of this model compare well with those of full simulations, suggesting that the simpler model captures the essential dynamics in an efficient way. 


\section{Introduction}

Network worms represent a serious threat to confidentiality, integrity, and availability of computer resources on the Internet. The existing automated network security solutions (e.g., antivirus software, firewalls, and intrusion detection systems) and human-dependent countermeasures (e.g., software patching, traffic blocking) have been deemed inadequate for timely detection and control of worm propagation $[6,34,35]$. Since the problem of network worms is worsening every year despite increasing efforts and expenditure on cyber-security [6], devising techniques for controlling their propagation is of great practical importance [22]. An important first step in developing control strategies is to understand (1) the dynamics of worm propagation and (2) how worm propagation is affected by the network structure.

Cyber-attacks employ malicious mobile code $(M M C)$ - a program designed to perform a malicious action by propagate copies of itself to computers, having a known vulnerability, on a network. MMCs can be classified into three broad categories based on the extent of human intervention required for their propagation [19], namely autonomous, human-dependent, and hybrid. Furthermore, based on their actions, MMCs may be grouped into three classes: Trojan horses, computer viruses, and network worms. A Trojan horse is a program that is given (fraudulently) the same name as a legitimate piece of software, but, when executed, it performs a malicious act. A computer virus is an MMC that modifies resident programs to perform malicious actions on a single computer. Like Trojan horses, computer viruses require human intervention to propagate on a network.

A network worm is a stand-alone program that propagates autonomously by sending copies of itself to other computers on the network. A computer is considered compromised if it hosts a replica of the worm. A worm propagates by sending a copy of autonomous MMC (AMMC) to a host, having the exploited vulnerability detected through scanning and probing. Therefore, the worm propagation is a random process that generates a complex system of interacting agents (AMMCs) over the propagation medium - a scale-free network. A recent study [38] has shown that: (1) probing attempts are in the range from one to three millions per day; (2) sources and destinations of intrusion are uniformly distributed among autonomous systems (AS); (3) scanning of ports other than 80 (HTTP), characteristic for Code Red and Nimda, constitute $40-80 \%$ of all intrusion attempts, and (4) while vertical and, in particular, horizontal scans are prevalent [29], other methods such as coordinated and stealthy scans are widely used.

The size of an AMMC is relatively small (less than 100 kilobyte), and the scan and probe pieces are negligibly small (one kilobyte and a dozen bytes, respectively) [27, $28,33]$. The code of Sapphire Worm, for example, consisted of only 376 bytes [20]. Thus, the only immediately observable effect of an attack on a network is an increase in the routing-related requests $[7,17]$, as the worm keeps probing different hosts. Due to the short time required for their propagation, worms can inflict considerable damage to the networks. For instance, Code Red worm [5] infected 150,000 computer systems in $14 \mathrm{~h}$. The damage inflicted by Nimda [4] to 86,000 computer systems has been estimated to USD 13 billion [6]. On January 24, 2003, the Sapphire Worm, taking advantage of a known vulnerability in Microsoft's SQL Server 2000, spread across different networks, including the Bank of America's network of 13,000 ATMs [20]. Since a malicious worm has the capacity for global impact on today's network-dependent society, developing models of propagation and control is a first step towards a comprehensive network security solution.

An important question in modelling worm propagation on a scale-free network
As the authors point out in their section 2, the Internet is well known to have a scale-free structure at several levels. This includes the network viewed as a collection of individual routers linked by transmission lines, or if the routers are lumped together in 'autonomous systems' (sub-networks of routers grouped under one administration, such as an Internet service provider). In either case, it has been found empirically that the degree distribution follows a power-law pattern; $p(k) \sim k^{-\gamma}$, where $p(k)$ is the probability of a node having degree $k$ and $\gamma$ is a positive constant. This implies that the Internet is very much unlike a simple random graph, in which the number of links per node does not fluctuate strongly from node to node. Yet such random graphs have formed the basis for most previous epidemiological studies of worm propagation, implying a clear need for studies on how the scale-free character of the real Internet alters traditional results.

It is this need that the authors attempt to address with their 'pair approximation' model, but before introducing it, they first review a number of different approximate models that have been applied to worm propagation in the past. As they point out, different dynamical models can be used to explore different questions. For example, one might treat nodes as having only two possible states, being either susceptible (S) or infected (I). This 'SI' model mimics an infection for which there are no available countermeasures, and so gives a kind of 'worst case' picture of the outcome. Alternatively, one might, for example, explore the slightly more detailed 'SIR' model, in which nodes can also be recovered (R), either as a result of some natural defence against the infection or some external intervention. This is useful for studying the effects of specific countermeasures that might fix worm infections or keep them isolated from specific nodes in the network. The authors also discuss a wide range of technical extensions and varia- 
is whether the population of computers (whose communication is captured by the network) is to be represented in groups based on average degree and risk of transmission or whether the computers and their communication are to be explicitly simulated. The latter level of detail provides a powerful tool as it allows the structure of the network to be included in the control techniques. Between the extremes of networks which are represented by a complete graph (see section 3) and individual-based simulation on scale-free networks, it is possible to divide the population of computers into groups with similar characteristics, e.g., degree (number of contacts), and derive a set of equations describing the propagation dynamics. Thus, the contact structure can be captured by stratifying the modelled population.

Our contribution here is a mathematical model of worm propagation that makes use of information about network structure order, size, degree distribution, and clustering coefficient (transitivity). We compare the results of the model with an individualbased simulation of worm propagation on scale-free graphs that model the Internet (obtained from the Oregon Route View project). We point out that, although the introduction of the Internet has arguably made the assumption of sparseness no longer valid, the idea of locality (especially in the case of analytical modelling of local propagation strategies) is still applicable, and, therefore, used in our model.

The paper is organized as follows: in section 2, we discuss adequate models for the propagation medium - the Internet, and define two abstractions of the Internet topology: the Microscopic Internet graph and the Macroscopic Internet graph. In section 3, we present a comprehensive survey of the existing models for worm propagation and control by (1) categorizing the models into seven classes and (2) identifying their advantages and disadvantages. Derivation of a pair-approximation susceptible-infectious-susceptible and susceptible-infectious-removed (SIR) model for worm propagation on scale-free graphs is presented in section 4. Finally, to test the accuracy of our approach, in section 5 we present a comparative empirical study of our pair-approximation model, the mean-field model, and the model of propagation on Erdös-Renyi graphs versus the results of the individual-based simulation.

\section{Adequate Model for the Propagation Medium}

Due to the diversity of exploited vulnerabilities, network worms can propagate on various types of networks. A (physical) network is a collection of interconnected computers, each with its distinct IP address. Such a network can be represented by a connected, undirected graph $G=(V$, $E)$, with the nodes as computers and the edges as the (physical) communication links (e.g., wire, optical cable). Since $G$ is connected, communication between any arbitrary pair of nodes $u$ and $v$, takes place through a $u, v$-path in $G$. Although graph $G$ just defined, represents a physical network, it may also represent a logical (or virtual) network. For example, in an e-mail graph, a node $v$ would represent a user and the (directed) edges emanating from $v$ would go to all the individuals in the e-mail address book of $v$. Likewise, in a webgraph each node $u$ would represent a web page and each (directed) edge emanating from $u$ would represent a hyperlink coming out of web page $u$.

Despite the differences in what they model, these graphs have the following similar characteristics: (1) degree distribution, (2) clustering coefficient, and (3) average distance. We note that a scale-free random graph model cannot be accepted as a valid representation of a real-world network if it satisfies only these three characteristics. To clarify these characteristics, we give the following definitions: tions of these models, each attuned to the study of some specific situation.

As Nikolski and colleagues point out, however, all previous studies of worm propagation suffer from one of two shortcomings. They have either been carried out on the assumption that the underlying network is a random graph (or some similarly unrealistic topology), or have made approximations that ignore the potentially significant correlations among the nodes in a network. The studies of reference 30, for example, assumed that the network has a scale-free structure and predicted the lack of any epidemic threshold in such a network, meaning that a worm, once introduced, should persist (possibly at low levels) no matter how low the probability of transmission between two nodes. This result conflicts, Nikolski et al. suggest, with the full simulations of their reference 13 , which did find a threshold. The difference in results may be attributable to reference 30 ignoring the correlations between nodes, by assuming, for example, that the neighbours of low-degree nodes will on average look like those of high-degree nodes. Instead, there may be correlations - highdegree nodes may tend to have low-degree neighbours, or vice versa, and such correlations could significantly influence the results.

Hence, in section 4 of the paper, the authors turn to their main project - to produce a set of simplified modelling equations that do take correlations into account, at least in some approximation. The basic variables for their model are the fractions of nodes that are in various states. In the SI model, for example, the two variables would be the fractions of susceptible and infected sites. To generalize slightly, here they consider the 'SIS' model in which an infected site becomes cured after a certain time, and hence becomes susceptible again. As they show in their equation (14), an expression for a change in the probabilities associated with one node involves the joint probabilities for pairs of adjacent nodes. 
Definition 1: The degree distribution gives the probability that a node, chosen uniformly at random, is of degreed.

Empirical studies of real-world networks have demonstrated that the degree distribution falls in the class of so-called scale-free (power-law) probability distributions, such that $P(d(v)=d)=d^{-f}(f$ is the exponent of the power-law degree distribution).

Definition 2: Given a graph $G=(V, E)$ and a node $v \in V$ of degree $d(v)$, the clustering coefficient $C_{v}$ of node $v$ is defined as the ratio between the total number of edges incident on all pairs of neighbours of $v$ and the number of edges in a clique formed by the neighbours of $v$.

The clustering coefficient of $G$ is the average of clustering coefficients over all nodes. The clustering coefficient of $G$ has values in the range $0 \leq C \leq 1$. There is yet another measure of clustering in graphs, called transitivity [21].

Definition 3: Transitivity is the ratio between the number of triangles and the total number of paths of length three.

Definition 4: Average distance of $G$ is the mean over all shortest distances between any connected nodes.

The Internet can be modelled on two levels: microscopic and macroscopic. In the Microscopic Internetgraph, nodes stand for routers and hosts, while edges represent communication links. The Macroscopic Internet graph can be thought of as a contraction of the Microscopic Internet graph: here, each node represents an AS (which incorporates a number of routers). To simplify the analysis, parallel edges and loops (having negligible influence in modelling propagation) will be deleted from the Macroscopic Internet graph. Two nodes in the Macroscopic Internet graph are adjacent if there is at least one pair of routers (belonging to different AS) that can communicate. Note that both, the Microscopic and the Macroscopic Internet graphs are undirected. Faloutsos et al. [10] studied both graphs, and concluded that the degree distribution follows a power law. In the Microscopic Internet graph, the exponent of the power law $f$ had a value of 2.48 , while in the Macroscopic Internet graph, the exponent ranged between $f=2.15$ and $f=2.2$ (studies were performed between 1997 and the end of 1998). Govindan and Tangmunarunkit [11] mapped the connectivity of nearly 150,000 router interfaces, confirming the power-law exponent of $f=2.3$. The studies of Yook et al. [39] conducted between 1997 and 1999 showed that the Macroscopic Internet graph has a clustering coefficient in the range from 0.18 to 0.3 and an average distance between 3.70 and 3.77 .

\section{Existing Models of Worm Propagation}

Due to the strong analogy between network worms and infectious diseases, epidemiological models have been widely used in modelling worm's propagation. Since a worm propagates along the edges of a network, we will use graph-theoretic terms to describe the existing epidemiological models of propagation. Epidemiological models are based on two simplifications [12]. (1) At any given time $t$, each node can be in one of a finite number of states, e.g. susceptible, quarantined-susceptible, removed-susceptible, infectious, quarantined-infectious, removed-infectious, and detected. The choice of which states to include in a model depends on the characteristics of the particular worm being analyzed and the purpose of the model. (2) Translation of the worm transmission mechanism into a probability that a node will infect another node. In a similar way, transitions between other states of the model are described by simple probabilities. Epidemiological models can be analyzed analytically or by means of simulation.

The propagation takes place on a graph $G$ with $n$ nodes and $m$ edges. Let $S(t)$ denote the number of susceptible nodes at time $t, Q_{S}(t)$ be the number of quaran-
Likewise, in equation (15), an expression for this joint probability inevitably involves a probability for three nodes, and so on, leading to an infinite hierarchy.

Obviously, this hierarchy presents a problem, as every attempt to 'flesh out' the equations introduces new and more complicated quantities. Even so, such hierarchies are well known in physics, for example, in the setting of the statistical mechanics of fluids or plasmas, and a general approach is to 'truncate' the hierarchy at some point by representing, say, a threepoint probability as a product of probabilities of lower-order, thereby achieving a closed system of equations. The authors do this in their equations (16-19), where they work in terms of the numbers of nodes rather than the probabilities. For example, $E\left(X_{a}\right)$ denotes the total number of nodes that have degree $a$ and are in state $X$ (either susceptible or infected), while $E\left(X_{a} Y_{b}\right)$ denotes the total number of pairs where one node, with degree $a$ and in state $X$, lies adjacent to a node of degree $b$ that is in state $Y$. They close the hierarchy in equation (18), by writing an approximation formula for a three-node quantity $E\left(X_{a} Y_{b} Z_{c}\right)$ in terms of two-node quantities and by using the concept of the 'transitivity' $\phi_{a b c}$, which is the ratio between the total number of triangles and the total number of paths of length three. The formulae in equation (19) all involve three-node quantities which can be approximated in terms of two-point quantities using equation (18), thereby yielding a set of equations that can be solved numerically and capture some element of the correlations in the network. [The authors' equation (20) simply gives the same results for the SIR model.]

Finally, in their section 5, the authors put these equations to the test. To do so, they compare the performance of their model results to two benchmarks - (1) a full-scale simulation of worm spreading on an Internet graph obtained from real data, and (2) an SIS model on two different networks (one in which every pair of nodes 
tined-susceptible nodes, $R_{S}(t)$ be the number of removed-susceptible nodes, $I(t)$ be the number of infectious nodes, $Q(t)$ be the number of quarantined-infectious, and $R(t)$ denote the number of removed nodes. The fraction of nodes in a particular state is represented by the lower case letter. Let $\beta$ denote the rate at which susceptible nodes are infected. Most models of propagation assume $\beta$ is constant, averaging out the differences in processor speed, network bandwidth, and location of the infectious node. The existing models also assume that a node cannot be infected multiple times.

\section{Susceptible-Infectious Model}

In this class of models, once a susceptible node becomes infectious, it does not change its state. These models can be used in the study of the worst-case propagation, when automated and human countermeasures are not available. Let the average degree of an infectious node be $\bar{d}$, and the fraction of infectious nodes at time $t$ be $i(t)$. The expected number of susceptible neighbours that can be infected by a given infectious node is $\bar{d}(1-i(t))$. Since there are $I(t)$ infectious nodes, the total rate of newly infected nodes is $\beta \bar{d}(1-i(t)) i(t)$. The general susceptible-infectious (SI) model is described by the differential equation (1):

$$
\frac{d i(t)}{d t}=\beta \bar{d}(1-i(t)) i(t),
$$

with boundary conditions:

$$
i(0)=\frac{I(0)}{n}>0
$$

and for all $t \geq 0, i(t)+s(t)=1$. The solution of equation (1) for the fraction of infectious nodes is the logistic curve:

$$
i(t)=\frac{i(0) e^{\beta^{\prime} t}}{1-i(0)+i(0) e^{\beta^{\prime} t}},
$$

where $\beta^{\prime}=\beta \bar{d}$. The $S$-shaped curve describing the fraction of infectious nodes has three regions: (1) slow start, when only few nodes are infected at every time step; (2) exponential growth, when the number of newly infected nodes grows exponentially, and (3) equilibrium state, when the number of infectious nodes assumes some value around which it fluctuates steadily.

If the worm propagates on the complete graph on $n$ nodes, $K_{n}$, where $\bar{d}=(n-1)$, the model (1) can asymptotically be written as:

$$
\frac{d i(t)}{d t}=\beta(1-i(t)) I(t),
$$

with boundary conditions:

$$
i(0)=\frac{I(0)}{n}>0
$$

and for all $t \geq 0, i(t)+s(t)=1$. One then has that

$$
i(t)=\frac{i(0) e^{\beta(n-1) t}}{1-i(0)+i(0) e^{\beta(n-1) t}} .
$$

Staniford et al. [27] applied model (2) to fit the data collected by the Chemical Abstracts Services from the propagation of Code Red I worm, and estimated the product $\beta(n-1)$ for Code Red I to be 1.8. However, they used the number of scanned nodes, which is much larger than the number of infectious nodes, thus, leading to erroneous conclusions. Weaver [33] and Wagner et al. [30] used this model to study four local propagation strategies: hit-list, topological, permutation, and local subnet, although we must note that the complete graph as underlying topology is inappropriate for studying such local strategies.

Zou et al. [41] used a modification of model (2) to analyze a trend-detection mechanism based on the traffic anomaly created by worms. The detection system is composed of distributed ingress and egress sensors for worm activity. When the monitoring system receives a surge of illegitimate scans, a Kalman filter is activated to estimate the parameter $\beta$. Since in the early stage the propagation exhibits exponential growth with constant, positive rate, the model can be described as are linked, and a random graph). The fullscale simulation should, in principle, represent the standard of accuracy, and as the data in figure 2 indicates, these simulations reveal realistic spreading behaviour. In a series of simulations, the authors found, unsurprisingly, that starting the infection off on a node of high degree reduces the time for it to spread throughout the network (it gets a head start with an opportunity to move quickly to many different points). Also, smaller networks get totally infected more rapidly than bigger ones. Less intuitively, they also find (see their fig. 3 ) that it is possible, in some cases, for a small increase in the infection rate to actually slow down the spread of the worm. The authors suggest quite plausibly that this is an effect of the correlations among nodes - infections spread the worm, but also reduce the number of new nodes that can be infected, potentially hindering the spread (at least partially).

The authors use their realistic Internet maps to derive some of the parameters in their pair-approximation model,including the important transitivities $\varphi_{a b c}$. As their results show (see fig. 5), their model gives excellent results in comparison with the simulations, and performs much more accurately than the other two approximate models. This success demonstrates the importance of modelling worm spreading using realistic network topologies. Moreover, it is not sufficient just to get the degree distribution of the topology right, but also to include correlations, which can have surprising consequences.

Mark Buchanan 


$$
I(t)=(1+\beta n d t) I(t-1) .
$$

The authors derived a bias-correction formula for estimation of the number of infectious nodes at time $t, I(t)$, from the number of observed infectious nodes $Z(t)$ : let $\sigma$ be the average number of scans sent by an infectious node. After time interval $d t$, the expected number of scans observed by $u$ monitors is $\beta u I(t) \sigma d t / 2^{32}$ (assuming the Internet is a complete graph), while the probability that any of the $I(t)-Z(t-1)$ infectious nodes are observed is 1 $\left(1-u / 2^{32}\right)^{\sigma d t}$. When the estimate of $\beta$ starts oscillating around a positive constant value, the worm has been detected. Yet, it is not evident how the topology might affect the detection mechanism of Zou et al.

In Erdös-Renyi random graphs with edge-density $p$, the expected degree of a node is $p(n-1)$. The propagation on these graphs can be described as:

$$
\frac{d i(t)}{d t}=\beta p(n-1)(1-i(t)) i(t),
$$

with solution:

$$
i(t)=\frac{i(0) e^{\beta p(n-1) t}}{1-i(0)+i(0) e^{\beta p(n-1) t}} .
$$

\section{SIS Model}

In this class of models, an infectious node recovers at some rate, and thus it becomes susceptible again. These models can be used in the study of worm's propagation when some computers are temporarily turned off but are not patched (e.g., the case of Code Red I worm). Let the average degree of an infected node be $\bar{d}$, and the rate at which an infectious node recovers be $\gamma$. The rate of newly infected nodes is proportional to the expected fraction of susceptible neighbours, the number of infected nodes, and the probability $\beta$. The rate at which infectious nodes recover is proportional to the number of infectious nodes and rate $\gamma$. The system of differential equation (4) describes the general SIS model:

$$
\frac{d i(t)}{d t}=\beta \bar{d}(1-i(t)) i(t)-\gamma i(t)
$$

with boundary conditions

$$
i(0)=\frac{I(0)}{n},
$$

and for all $t \geq 0, i(t)+s(t)=1$. From equation (4),

$$
\frac{d i(t)}{d t}<0
$$

if and only if

$$
s(t)<\frac{\gamma}{\beta \bar{d}}=\delta .
$$

Thus, the worm 'dies out' if the initial fraction of susceptible nodes is below the epidemic threshold

$$
\frac{\gamma}{\beta \bar{d}} \text {. }
$$

The solution of equation (4) gives a functional form for the fraction of infectious nodes:

$$
i(t)=\frac{(1-\delta) i(0)}{i(0)+(1-\delta-i(0)) e^{-\left(\beta^{\prime}-\gamma\right) t}},
$$

where $\beta^{\prime}=\beta \bar{d}$. If the worm propagates on the complete graph on $n$ nodes, $K_{n}$, where $\bar{d}=(n-1)$, the model (4) can asymptotically be written as [34]:

$$
\frac{d i(t)}{d t}=\beta(1-i(t)) I(t)-\gamma i(t),
$$

with solution

$$
i(t)=\frac{(1-\delta) i(0)}{i(0)+(1-\delta-i(0)) e^{-(\beta(n-1)-\gamma) t}} .
$$

Solomon [26] studied a modification of model (5) where the rate $\gamma$ is a weighted average of the rate $\gamma_{1}$ (for computers not running antivirus software), applicable to the fraction of infectious nodes, and the rate $\gamma_{2}$ (for computers running the most recent version of antivirus software), applicable to the fraction of susceptible nodes, i.e., $\gamma=\gamma_{1} i(t)+\gamma_{2}(1-i(t))$. With this modification Solomon found that the necessary effectiveness of the anti-virus software (described by the rate $\gamma$ ) should be 0.5 in order to stop the propagation before it achieves exponential growth.

Kephart and White [14] and Kephart [15] employed model (4) to study the effects of three topologies on the propagation of viruses: Erdös-Renyi random graphs, regular lattices of degree 8 , and hierarchical random graphs. For the ErdösRenyi random graphs with $\bar{d} \geq 5$, simulation results coincide with the predictions of the model. The simulation study of propagation on 100-by-100 lattice demonstrates quadratic growth, in contrast with the exponential growth characteristics for the complete graph and Erdös-Renyi graphs. The hierarchically clustered random graphs in this study are generated as follows: given a rooted tree of height $h$, in which every node has a degree $(d+1$; i.e., it has $d$ successors), the nodes of the graph are the leaves of the tree. Two nodes, $u$ and $v$, are made adjacent with probability $P(h(w))$ proportional to the height of node $w$ - the first common ancestor of nodes $u$ and $v$. In his simulation, Kephart used $P(h(w))=\alpha p^{h(w)}$, where parameter $p$ is used to control the degree of localization (when $p$ tends to 0 , the graph is composed of isolated nodes, while when $p$ approaches 1 , the topology of the hierarchically clustered random graph is asymptotically that of the Erdös-Renyi random graphs). Here, the propagation shows subexponential growth. Further simulation studies conducted by Kephart [15] show that sparsely connected (random) graphs inhibit the propagation.

Previous models are limited in their accuracy due to their simplistic treatment of timing factors, such as infection delay the length of time between the instant of the worm's arrival at a node and the instant when this node becomes infectious to its neighbours. Model (4) could be altered to incorporate the infectious delay, as follows [32]: 


$$
\frac{d i(t)}{d t}=\beta \bar{d} e^{-\gamma \varepsilon}(1-i(t)) i(t-\varepsilon)-\gamma i(t),
$$

where $i(t-\varepsilon)=0$ for $t<\varepsilon$. At time $t \geq \varepsilon$, the fraction of infectious nodes is the same as the fraction of infectious nodes at time $(t-\varepsilon)$, since all nodes infected between $(t-\varepsilon)$ and $t$ are delayed. The term $e^{-\gamma}$ accounts for the transfer of a node from infectious to susceptible state during the delay period. Equation (6) belongs to the class of non-linear delayed differential equations, which can be solved under the assumption $i(t-\varepsilon)=i(t)$. Wang and Wang [32] support their analytical solution with simulation similar to that of Kephart and White [14], and show that the epidemic threshold depends not only on the average degree, but also on the infection delay. In addition, Kim et al. [16] performed a simulation study of the propagation on a subgraph of the Internet, using a constant delay equal to the average round-trip time obtained from real-life traffic.

Pastor-Satorras and Vespignani [23] modified model (4) to study the effects of the scale-free Barabasi-Albert topology on the propagation with rate of recovery $\gamma=$ 1 . Since a scale-free degree distribution is not concentrated around its mean value, the model must include differential equation for every group of nodes of degree $k$ :

$$
\begin{aligned}
& \frac{d i_{k}(t)}{d t}= \\
& \beta k\left(1-i_{k}(t)\right) \Theta\left(\left\{i_{k}(t)\right\}_{d_{\min }}^{d_{\max }}\right)-i_{k}(t),
\end{aligned}
$$

where

$$
\Theta\left(\left\{i_{k}(t)\right\}_{k=d_{\min }}^{d_{\max }}\right)
$$

describes the probability that a susceptible node of degree $k$ is adjacent to an infectious node. For a scale-free network, the probability that an edge is incident on a node of degree $k$ is $k P(k) / \bar{d}$. The average probability that an edge is incident on an infectious node is then

$$
\Theta(t)=\frac{1}{\bar{d}} \sum_{k=d_{\min }}^{d_{\max }} k P(k) i_{k}(t) .
$$

The conclusion of this model is that scale-free topologies do not have epidemic threshold. The authors also argued that the cutoff in the scale-free distribution forces a non-zero epidemic threshold. We point out that the result of this study is limited to scale-free topologies without degree correlations. Contrary to this result, the simulation study of Eguiluz and Klemm [9] demonstrates that in the so-called structured scale-free networks, where adjacent nodes share a large number of common neighbours, there exists a non-zero threshold even in the limit of large $n$.

While the results of the presented studies are valuable, a model, where nodes that have recovered and are no longer susceptible, could better approximate the realistic propagation of a worm when human countermeasures are in place.

\section{SIR Model and Its Variations}

In this class of models, an infectious node can be removed (i.e., it can no longer spread the worm). This model can be used to study the effects of software patching and traffic blocking. At any time $t$, a node can be susceptible, infectious, or removed. Let $\gamma$ be the rate at which infectious nodes are removed. Using analogous arguments as in the previous section, the general SIR model can be written as:

$$
\begin{aligned}
& \frac{d i(t)}{d t}=\beta \bar{d}(1-i(t)) i(t)-\gamma i(t), \\
& \frac{d r(t)}{d t}=\gamma i(t),
\end{aligned}
$$

with boundary conditions:

$$
i(0)=\frac{I(0)}{n} \geq 0, r(0)=\frac{R(0)}{n} \geq 0,
$$

and for all $t \geq 0, i(t)+s(t)+r(t)=1$. The epidemic threshold for SIR models is analogous to the one in SIS models. Zou et al. [40] used a modification of the system (8) to determine the effect of human countermeasures (on removing both susceptible and infectious nodes) and the decreasing rate $\beta(t)$. This so-called two-factor model assumes complete graph as underlying topology, and a constant fraction of the removed-infectious nodes at any time $t$ :

$$
\begin{aligned}
& \frac{d i(t)}{d t}= \\
& \beta(t)\left(1-r(t)-r_{s}(t)-i(t)\right) i(t)-\frac{d r(t)}{d t}, \\
& \frac{d r(t)}{d t}=\gamma i(t), \\
& \frac{d r_{s}(t)}{d t}= \\
& \mu\left(1-r(t)-r_{s}(t)-i(t)\right)(r(t)+i(t)), \\
& \beta(t)=\beta(0)(1-i(t))^{\eta} .
\end{aligned}
$$

It is unclear, however, how the parameters have been chosen in order to fit the data from the Code Red I worm propagation.

Boguna et al. [2] studied the SIR model, with the probability $\gamma=1$, on scale-free topologies. Using the notation introduced in the previous subsection, the model can be formulated as follows:

$$
\begin{aligned}
& \frac{d i_{k}(t)}{d t}=\beta k\left(1-i_{k}(t)\right) \Theta\left(\left\{i_{k}(t)\right\}_{d_{\min }}^{d_{\max }}\right)-i_{k}(t), \\
& \frac{d r_{k}(t)}{d t}=\gamma i_{k}(t),
\end{aligned}
$$

which can be solved if one assumes that $i(0)$ is very small at the beginning of the propagation. Pastor-Satorras and Vespignani [23] conducted a simulation study to investigate the effects of node immunization (i.e. node removal) on the propagation, before the worm is introduced in the network. They demonstrated that random immunization is inefficient in slowing down the propagation; however, immunization targeted at nodes of highest degrees can significantly inhibit the growth of propagation. While the latter result seems interesting, the authors argue that detecting nodes of high degrees in scale-free networks is a difficult problem.

Similarly, the simulation study of Wang et al. [31] examines the effects of immunization of nodes on the propagation on two topologies: rooted trees and clustered networks (composed of cliques interconnected with a small number of edges). The simulation's parameter is the propagation fan-out, the number of nodes to which the worm can 
send replicas at each time step. The time needed for the worm to propagate from one node to another is assumed to be one time tick. The first set of simulation is conducted on networks where no immunized nodes exist to determine the number of times a node is re-infected (called re-infection count). Two types of immunization are simulated - random and selective. Random immunization performs better on rooted trees as there is only one path between any two nodes; thus, it is possible to cut off an entire subtree of the network, which is not the case with the clustered network. For the case-selective immunization in rooted trees, nodes with highest re-infection counts were chosen (note, these nodes coincide with nodes with largest degrees). In the case of clustered networks, two strategies are used:first based on the re-infection count, and second on the weighted sum of the inter-cluster and inner-cluster degrees for every node. The first strategy was able to contain the propagation, but results in a higher propagation rate. The second could slow down the propagation rate, but was unable to contain the propagation.

The principal disadvantage of the studies by Wang et al. [31] and Pastor-Satorras and Vespignani [23] is that immunization is static, i.e., a fraction of nodes is immunized before the worm starts propagating. In reality, the countermeasures should be dynamic in nature to play an important role in slowing down the propagation of the worm.

\section{Susceptible-Infectious-Detected-} Removed (SIDR) Model

This model was analyzed by Williamson and Leveille [37] in order to determine the effectiveness of the behaviour-blocking approach called virus throttling [36]. Virus throttling is an automatic mechanism for slowing a worm's propagation. Here, a node can be in one of the four states: susceptible, infectious, detected (in which the virus has been detected and cannot actively spread further), and removed. The model assumes complete graph as underlying topology. The model involves two stages: in the first stage, prior to the release of the virus signature, nodes progress from the susceptible to the infectious state at some rate $\beta$. In the second stage, after some time from the start of the propagation, the virus is detected at some rate $\gamma$. Two quantities are studied: the number of infectious nodes and the duration of propagation. The model incorporates virus throttling by dividing the nodes into two groups - throttled and unthrottled. If a throttled node is infected, it does not spread the virus, and immediately enters the detected state. The results of this study show that when more than half of the nodes have throttles, even a late signature will result in a small outbreak.

\section{Susceptible-Infectious-Removed-} Susceptible (SIRS) Model

Wang and Wang [32] used a modification of SIS model (4) to study the node's vigilance against infection. Once an infectious node is removed, it remains in this state for a length of time $v$, called vigilance period, after which the removed node becomes susceptible again. Here, the susceptibility of a node is modelled via a parameter $\Phi$ that takes values between 0 (indicating complete susceptibility) and 1 (indicating immunity). The model is described by the non-linear delay differential equation (11):

$\frac{d i(t)}{d t}=\beta \bar{d}\left(1-i(t)-\int_{t-\nu}^{t} i(t)\right) i(t)-\gamma i(t)(11)$

whose solution shows that the number of infectious nodes decreases as the vigilance period increases. It is worth noting the node's vigilance has no impact on the epidemic threshold.

\section{Compartmental Epidemiological Models}

Compartmental epidemiological models are used with stratified population. The topology in this model is the Macroscopic Internet graph, where every node represents a dense region - an AS. These models can be used to study intra-AS propagation, with the assumption that within an AS (with $n_{j}$ nodes) the worm propagates as on a complete graph $K_{n_{j}}$. The infectious attempts can then be modelled as being external or internal to an AS. If the macroscopic Internet graph has $k$ nodes, the SI compartmental model can be written as:

$$
\frac{d i_{j}(t)}{d t}=\left[\sum_{l=1}^{k} \beta \frac{n_{l}}{N} i_{l}(t)\right]\left(1-i_{j}(t)\right),
$$

where $1 \leq j \leq k$. Here, the parameter $N$ denotes the total number of IP addresses. Serazzi and Zanero [25] used model (12) to derive equations for the bandwidth consumption at each node. For the SIR compartmental model, Liljenstam et al. [18] obtained:

$$
\begin{aligned}
\frac{d i_{j}(t)}{d t} & =\left[\sum_{l=1}^{k} \beta \frac{n_{l}}{N} i_{l}(t)\right]\left(1-i_{j}(t)\right)-\gamma i_{j}, \\
\frac{d r_{j}(t)}{d t} & =\gamma i_{j}(t),
\end{aligned}
$$

where, again, $1 \leq j \leq k$. Liljenstam et al. [18] used model (13) to study the destabilizing effects of worm propagation on the network infrastructure, since the compartmental approach allows for inclusion of limited details about communication protocols. In this simulation study, the scan traffic is modelled by using a combination of the average scan rate, individual infection rates, and size of address space for each AS.

\section{Discrete-Time Approximation Models}

Chen et al. [3] developed a deterministic approximation model of propagation on a complete graph $K_{n}$. If $\sigma$ is the average scanning rate, with the assumption that the total number of nodes is $2^{32}$, the average number of newly infected nodes at step $(t+1)$ is $(S(t)-I(t))\left[1-\left(1-1 / 2^{32}\right)^{\sigma I(t)}\right]$. If the probability of removal is $\gamma$, in the next time step $\gamma I(t)$ nodes will become 
susceptible. Thus, the propagation can be described by a system of recurrences for the number of infectious and susceptible nodes.

\section{Pair-Approximation Model on Scale-Free Networks}

The existing epidemiological models on scale-free graphs $[23,40]$ do not explicitly give the system of differential equations for the propagation dynamics. The comparative studies include either simulation of worm's propagation on a macroscopic level or a system of differential equation for propagation on Erdös-Renyi and regular graphs. Thus, in all models described in section 3, it is not evident how a realistic, scale-free network structure might affect the worm propagation.

Worm propagation is a random process that takes place on networks, such as the Internet, World Wide Web, and e-mail network, modelled as large scale-free random graphs. Using the salient features of the underlying scale-free graphs, here, we develop a realistic model of worm's propagation and techniques for dynamic quarantining. Cast in the SI framework, our model can be used to study the worst-case propagation and determine the optimal time for undertaking preventive action. On the other hand, cast in the SIR framework, this model can be used in the study of quarantining techniques against network worms.

Our model of worm's propagation belongs to the class of pair-approximation network models. The benefit of this class of models is that it can incorporate the spatial structure that the existing epidemiological models of propagation ignore. A survey of pair-approximation models is given by Rand [24].In the pair-approximation model, the variables are the fractions of pairs of nodes in certain states. Usually, these equations contain higher-order correlations (e.g., triples of nodes in certain states) which are approximated by the lower-order correlations. For the most part, previous work on pair-approximation models de-

scribes processes on regular lattices. Our model extends the work by Earnes and Keeling [8] (for triangle-free networks) and Bauch [1] (for dynamic partnerships), and makes pair-approximation applicable to various scale-free topologies.

Next, we present the derivation of the system of differential equations describing the propagation in the SIS framework. Let $N(u)$ be the neighbourhood of a node $u$, $p_{t}\left(i_{u}\right)$ the probability that, at time $t$, node $u$ is infectious, and let $p_{t}\left(s_{u}, i_{v}\right)$ be the joint probability that two adjacent nodes $u$ and $v$ are susceptible and infectious, respectively. The time evolution of the state of a single node in the SIS epidemic process can be written in the following form:

$\frac{d p_{t}\left(i_{u}\right)}{d t}=\beta \sum_{v \in N(u)} p_{t}\left(s_{u}, i_{v}\right)-\gamma p_{t}\left(i_{u}\right)$,
$p_{t}\left(s_{u}\right)+p_{t}\left(i_{u}\right)=1$.

One can also develop an equation for the time evolution of $p_{t}\left(s_{u}, i_{v}\right)$, which in turn involves higher-order correlations. To solve this problem, we resort to some approximation scheme: for instance, the SIS epidemiological model assumes that $p_{t}\left(s_{w}, i_{v}\right)=$ $p_{t}\left(s_{u}\right) p_{t}\left(i_{v}\right)$, and thus neglects correlation between states of nodes. In our approach, $p_{t}\left(i_{u}\right)$ and $p_{t}\left(s_{u}, i_{v}\right)$ are kept as variables of interests while the higher-order correlations are expressed, via some appropriate approximation, in terms of these quantities. The time evolution of $p_{t}\left(s_{u}, i_{v}\right)$ can be derived by using the Kolmogorov forward equation:

$$
\begin{aligned}
& \frac{d p_{t}\left(s_{u}, i_{v}\right)}{d t}= \\
& -(\beta+\gamma) p_{t}\left(s_{u}, i_{v}\right)-\beta \sum_{w \in N(u)-v} p_{t}\left(i_{w}, s_{u}, i_{v}\right. \\
& +\beta \sum_{w \in N(v)-u} p_{t}\left(s_{u}, s_{v}, i_{w}\right)+\gamma p_{t}\left(i_{u}, i_{v}\right) .
\end{aligned}
$$

Let $\Lambda_{a}$ be the set of integers, representing the degrees of the neighbours for all nodes of degree $a$, and $[a b]$ be the number of edges incident on nodes of degrees $a$ and $b$. Furthermore, let $X, Y$, and $Z$ represent a state of a node (e.g., susceptible and infectious).
Given a node $u$ of degree $a$ and a node $v$ of degree $b$, define

$$
P_{t}\left(X_{a}, Y\right)=\frac{1}{a} \sum_{d(u)=a, d(v) \in \Lambda_{a}} P_{t}\left(x_{u}, y_{v}\right),
$$

and

$$
\begin{aligned}
& P_{t}\left(X_{a}, Y_{b}, Z\right)= \\
& \frac{1}{[a b]} \sum_{d(u)=a, d(v)=b, d(w) \in \Lambda_{b}} P_{t}\left(x_{u}, y_{v}, z_{w}\right) .
\end{aligned}
$$

Remark:

$$
P_{t}\left(X_{a}, Y\right)=\sum_{k \in \Lambda_{a}} P_{t}\left(X_{a}, Y_{k}\right)
$$

and

$$
P_{t}\left(X_{a}, Y_{b}, Z\right)=\sum_{k \in \Lambda_{b}} P_{t}\left(X_{a}, Y_{b}, Z_{k}\right) .
$$

Let $E\left[X_{a}\right]$ denote the number of nodes of degree $a$ in state $X, E\left[X_{a} Y_{b}\right]$ the number of pairs of nodes of degree $a$ in state $X$, adjacent to nodes of degree $b$ in state $Y$, and $E\left[X_{a} Y_{b} Z_{c}\right]$ denote the number of triples where a node of degree $b$ in state $Y$, is adjacent to a node of degree $a$ and a node of degree $c$, in state $X$ and $Z$, respectively. By multiplying equation (14) by $n_{a}$, the number of nodes of degree $a$ in the graph $G$, one can obtain the following equation:

$$
\frac{d E\left[I_{a}\right]}{d t}=\beta \sum_{k \in \Lambda_{a}} E\left[S_{a} I_{k}\right]-\gamma E\left[I_{a}\right],
$$

where $E\left[S_{a}\right]+E\left[S_{b}\right]=n_{a}$. Similarly, one can transform equation (15) to obtain:

$$
\begin{aligned}
\frac{d E\left[S_{a} I_{b}\right]}{d t}= & -(\beta+\gamma) E\left[S_{a} I_{b}\right]-\beta \sum_{k \in \Lambda_{a}} E\left[I_{k} S_{a} I_{b}\right] \\
& +\beta \sum_{k \in \Lambda_{b}} E\left[S_{a} S_{b} I_{k}\right]+\gamma E\left[I_{a} I_{b}\right] . \quad \text { (17) }
\end{aligned}
$$

Let $\varphi_{a b c}$ denote the transitivity among nodes of degree $a, b$, and $c$, i.e., the ratio of the number of 3 cycles to the number of connected triples whose nodes are of degree $a, b$, and $c$. To approximate the third moment $E\left[X_{a} Y_{b} Z_{c}\right]$, one can use the definition of multiplicative moments of two variables $[17,32]$ and the transitivity $\varphi_{a b c}$, to find: 
$E\left[X_{a} Y_{b} Z_{c}\right]=\frac{b-1}{b} \frac{E\left[X_{a} Y_{b}\right] E\left[Y_{b} Z_{c}\right]}{E\left[Y_{b}\right]}\left(\left(1-\varphi_{a b c}\right)+\varphi_{a b c} \frac{n^{2}}{2 m} \frac{E\left[X_{a} Z_{c}\right]}{E\left[X_{a}\right] E\left[Z_{c}\right]}\right)$

Similarly, one can derive formulae for the other second moments and appropriate approximation of the third moments to obtain the following pair-approximation for the SIS framework:

$$
\begin{aligned}
& \frac{d E\left[I_{a}\right]}{d t}=\beta \sum_{k \in \Lambda_{a}} E\left[S_{a} I_{k}\right]-\gamma E\left[I_{a}\right], \\
& \frac{d E\left[S_{a}\right]}{d t}=\gamma E\left[I_{a}\right]-\beta \sum_{k \in \Lambda_{a}} E\left[S_{a} I_{k}\right], \\
& \frac{d E\left[S_{a} S_{b}\right]}{d t}=-\beta\left(\sum_{k \in \Lambda_{a}} E\left[I_{k} S_{a} S_{b}\right]+\sum_{k \in \Lambda_{b}} E\left[S_{a} S_{b} I_{k}\right]\right)+\gamma\left(E\left[S_{a} I_{b}\right]+E\left[I_{a} S_{b}\right]\right) \\
& \frac{d E\left[S_{a} I_{b}\right]}{d t}=-(\beta+\gamma) E\left[S_{a} I_{b}\right]-\beta\left(\sum_{k \in \Lambda_{a}} E\left[I_{k} S_{a} I_{b}\right]-\sum_{k \in \Lambda_{b}} E\left[S_{a} S_{b} I_{k}\right]\right)+\gamma E\left[I_{a} I_{b}\right], \\
& \frac{d E\left[I_{a} I_{b}\right]}{d t}=\beta\left(E\left[S_{a} I_{b}\right]+E\left[I_{a} S_{b}\right]+\sum_{k \in \Lambda_{a}} E\left[I_{k} S_{a} I_{b}\right]+\sum_{k \in \Lambda_{b}} E\left[I_{a} S_{b} I_{k}\right]\right)-2 \gamma E\left[I_{a} I_{b}\right] .
\end{aligned}
$$

Now, the system of the differential equations (19) can be numerically solved by using the approximation given in equation (18). Note that our model differs from the one presented in Bauch [1] and Earnes and Keeling [8] since we take into consideration the transitivity $\varphi_{a b c}$, which turns out to have a significant effect on the outcome of the model (see section 5).

Model (19) can be altered to obtain the system of differential equations (20), describing the dynamics of propagation in the SIR framework:

$$
\begin{aligned}
& \frac{d E\left[I_{a}\right]}{d t}=\beta \sum_{k \in \Lambda_{a}} E\left[S_{a} I_{k}\right]-\gamma E\left[I_{a}\right], \\
& \frac{d E\left[S_{a}\right]}{d t}=-\beta \sum_{k \in \Lambda_{a}} E\left[S_{a} I_{k}\right], \\
& \frac{d E\left[R_{a}\right]}{d t}=\gamma E\left[I_{a}\right], \\
& \frac{d E\left[S_{a} S_{b}\right]}{d t}=-\beta\left(\sum_{k \in \Lambda_{a}} E\left[I_{k} S_{a} S_{b}\right]+\sum_{k \in \Lambda_{b}} E\left[S_{a} S_{b} I_{k}\right]\right), \\
& \frac{d E\left[S_{a} I_{b}\right]}{d t}=-(\beta+\gamma) E\left[S_{a} I_{b}\right]-\beta\left(\sum_{k \in \Lambda_{a}} E\left[I_{k} S_{a} I_{b}\right]-\sum_{k \in \Lambda_{b}} E\left[S_{a} S_{b} I_{k}\right]\right), \\
& \frac{d E\left[S_{a} R_{b}\right]}{d t}=-\beta \sum_{k \in \Lambda_{a}} E\left[I_{k} S_{a} R_{b}\right]+\gamma E\left[S_{a} I_{b}\right], \\
& \frac{d E\left[I_{a} I_{b}\right]}{d t}=\beta\left(E\left[S_{a} I_{b}\right]+E\left[I_{a} S_{b}\right]+\sum_{k \in \Lambda_{a}} E\left[I_{k} S_{a} I_{b}\right]+\sum_{k \in \Lambda_{b}} E\left[I_{a} S_{b} I_{k}\right]\right)-2 \gamma E\left[I_{a} I_{b}\right], \\
& \frac{d E\left[I_{a} R_{b}\right]}{d t}=\beta \sum_{k \in \Lambda_{a}} E\left[I_{k} S_{a} R_{b}\right]+\gamma\left(E\left[I_{a} I_{b}\right]-E\left[I_{a} R_{b}\right]\right) .
\end{aligned}
$$

\section{Pair-Approximation Model versus Individual-Based Simulation}

Our goal is to test the accuracy of the pair-approximation model (19) in comparison to: (1) the individual-based simulation of the worm propagation on a Macroscopic Internet graph (on $n$ nodes and average degree $\bar{d}$ ), and (2) the standard SIS model (which ignores correlation) on two topologies: the complete graph on $n$ nodes (model (5)) and the Erdös-Renyi graph on $n$ nodes with average degree $\bar{d}$ (model (4)).

\begin{tabular}{|c|r|r|}
\hline Date & \multicolumn{1}{|c|}{ Order } & \multicolumn{1}{c|}{ Size } \\
\hline \hline 08.11 .1997 & 3015 & 5156 \\
\hline 02.04 .1998 & 3522 & 6324 \\
\hline 03.07 .1998 & 3797 & 6936 \\
\hline 02.10 .1998 & 4180 & 7768 \\
\hline 14.01 .1999 & 4517 & 8376 \\
\hline 02.04 .1999 & 4885 & 9276 \\
\hline 02.07 .1999 & 5357 & 10328 \\
\hline 02.10 .1999 & 5861 & 11313 \\
\hline 02.01 .2000 & 6474 & 12572 \\
\hline 03.04 .2000 & 7246 & 14629 \\
\hline 02.07 .2000 & 7956 & 15943 \\
\hline 02.10 .2000 & 8836 & 17823 \\
\hline 02.01 .2001 & 9048 & 18172 \\
\hline 16.03 .2001 & 10515 & 21455 \\
\hline
\end{tabular}

Fig. 1. Macroscopic Internet graphs used in simulations.

The empirical study is conducted on Macroscopic Internet graphs. To obtain the Macroscopic Internet graphs, we used the data for interconnectedness of the Internet on the AS level collected by the University of Oregon Route View Project and made available by NLANR (National Laboratory of Applied Network Research). We considered snapshots of the Internet of various orders and sizes, shown in figure 1. The preprocessing step consists of determining parameters for model (19): for given degrees $a, b$ and $c$, the number of adjacent nodes of degree $a$ and $b$, the set $\varphi_{a}$, and the transitivity $\Lambda_{a b c}$ are determined.

Next, we developed an individualbased simulation of the stochastic propagation process on a Macroscopic Internet graph. The individual-based simulation has two advantages: First, the propagation process and the underlying topology can be controlled to simulate different scenarios. Second, this simulation provides very precise and detailed information about the propagation dynamics without any biases which might be present in real data. The individual-based simulation combines Monte Carlo simulation of events, taking place at given rates, with an event scheduler that deter- 


\begin{tabular}{|r|r|r|r|r|r|r|}
\hline \multirow{2}{*}{ AS graph 02.10.1998 } & \multicolumn{5}{|c|}{ beta } \\
\cline { 3 - 7 } & & 0.2 & 0.5 & 0.9 & 1.5 & 1.8 \\
\hline min degree & node & & & & & \\
\hline 1 & 47 & 18.3675 & 7.89627 & 4.74167 & 3.20504 & 3.55996 \\
\hline 2 & 17 & 18.0627 & 7.91097 & 4.6643 & 3.19542 & 3.46717 \\
\hline 3 & 22 & 18.4302 & 7.76855 & 4.69197 & 3.14913 & 3.33457 \\
\hline max degree & node & & & & & \\
\hline 590 & 5 & 17.158 & 7.73703 & 4.45511 & 3.01981 & 3.34582 \\
\hline 524 & 12 & 16.9676 & 7.37089 & 4.50048 & 2.96612 & 3.39495 \\
\hline 355 & 10 & 17.2982 & 7.48106 & 4.5871 & 3.10994 & 3.2987 \\
\hline
\end{tabular}

$1 \mathrm{pt}$

\begin{tabular}{|c|c|c|c|c|c|c|}
\hline \multirow{2}{*}{\multicolumn{2}{|c|}{ AS graph 02.07.1999 }} & \multicolumn{5}{|c|}{ beta } \\
\hline & & 0.2 & 0.5 & 0.9 & 1.5 & 1.8 \\
\hline min degree & node & & & & & \\
\hline 1 & 63 & 18.3734 & 7.98559 & 4.81368 & 3.24062 & 3.42445 \\
\hline 2 & 19 & 18.5593 & 8.17793 & 4.70665 & 3.19895 & 3.48956 \\
\hline 3 & 15 & 18.5207 & 7.93869 & 4.74685 & 3.18794 & 3.343 \\
\hline max degree & node & & & & & \\
\hline 1193 & 2 & 17.4871 & 7.43152 & 4.44526 & 2.98155 & 3.39874 \\
\hline 674 & $\overline{10}$ & 17.6827 & 7.58919 & 4.57659 & 3.11 & 3.41901 \\
\hline 588 & 7 & 17.5386 & 7.70924 & 4.63347 & 3.10909 & 3.38447 \\
\hline
\end{tabular}

$1 \mathrm{pt}$

\begin{tabular}{|r|r|c|c|c|c|c|}
\hline \multicolumn{2}{|c|}{ AS graph 02.07.2000 } & \multicolumn{5}{|c|}{ beta } \\
\hline min degree & node & 0.2 & 0.5 & 0.9 & 1.5 & 1.8 \\
\hline 1 & 15 & 19.8676 & 8.42701 & 5.11502 & 3.42114 & 3.71483 \\
\hline 2 & 18 & 20.1391 & 8.62913 & 5.15767 & 3.41545 & 3.56204 \\
\hline 3 & 23 & 19.4788 & 8.44671 & 5.05909 & 3.3409 & 3.5603 \\
\hline max degree & node & & & & & \\
\hline 1772 & 2 & 18.9615 & 8.0676 & 4.97258 & 3.29313 & 3.48871 \\
\hline 961 & 9 & 19.2357 & 8.28964 & 4.90184 & 3.33247 & 3.49154 \\
\hline 802 & 7 & 18.9133 & 8.24647 & 4.96538 & 3.34996 & 3.47815 \\
\hline
\end{tabular}

$1 \mathrm{pt}$

\begin{tabular}{|r|r|r|c|c|c|c|}
\hline \multicolumn{2}{|c|}{ AS graph 16.03.2001 } & \multicolumn{5}{|c|}{ beta } \\
\hline min degree & node & 0.2 & 0.5 & 0.9 & 1.5 & 1.8 \\
\hline 1 & 44 & 21.0673 & 8.91663 & 5.35035 & 3.57629 & 3.76316 \\
\hline 2 & 37 & 21.385 & 8.90863 & 5.35318 & 3.47916 & 3.74718 \\
\hline 3 & 34 & 20.6299 & 8.93965 & 5.33861 & 3.54236 & 3.64725 \\
\hline max degree & node & & & & & \\
\hline 2277 & 2 & 20.1728 & 8.44475 & 4.98278 & 3.37857 & 3.58247 \\
\hline 1231 & 13 & 20.3132 & 8.67817 & 5.248 & 3.46303 & 3.62322 \\
\hline 899 & 15 & 20.4644 & 8.87768 & 5.2025 & 3.42517 & 3.69419 \\
\hline
\end{tabular}

Fig. 2. Time to propagate to all nodes of a Macroscopic Internet graph for five different values of the parameter $\beta$.

mines the order in which events happen in the system. The scheduler is implemented as a priority queue. The system is composed of nodes that can be either susceptible or infectious. There are two types of events that can take place: infection and curing. If a node $u$ is infectious, it attempts infection of each of its neighbours at rate $\beta$. Node $u$ might be cured, and, thus, become susceptible, at rate $\gamma$. Let us assume that $u$ has been cured at time $t$, and has been re-infected at time $t+d t$. Any infection generated by the node $u$ in the time interval $[t, t+d t]$ can be discarded by the scheduler. The event of $u$ attempting infection of an already infectious neighbour $v$ at time $t$ is also discarded by the scheduler.
We simulated worm propagation in the susceptible-infectious framework in order to determine the time the worm takes to infect all nodes of a given graph $G$. Simulations were performed on ten Macroscopic Internet graphs (the results for four graphs, identified by the top entry in the left-most column appear in fig. 2). To determine how choice of the initial node influences the propagation, we first determined the labels of three nodes with the smallest degrees and three nodes with the highest degree, shown in the first and second column of each table in figure 2. The rest of the entries show the average time over 100 simulations for the worm to propagate on all nodes by starting from a prespecified initial node and spreading with infectious rate $\beta$. The general results of the experiments can be summarized as follows:

1) The time to propagate to all nodes decreases with the increase of the degree of the initial node. As an infectious node of a higher degree has a bigger pool of susceptible nodes, it gives the worm the possibility to establish a considerable fraction of infectious nodes in the early stages of the propagation. On average, the propagation to all nodes of $G$ initiated from a node of maximum degree takes time which is by $5 \%$ shorter compared to

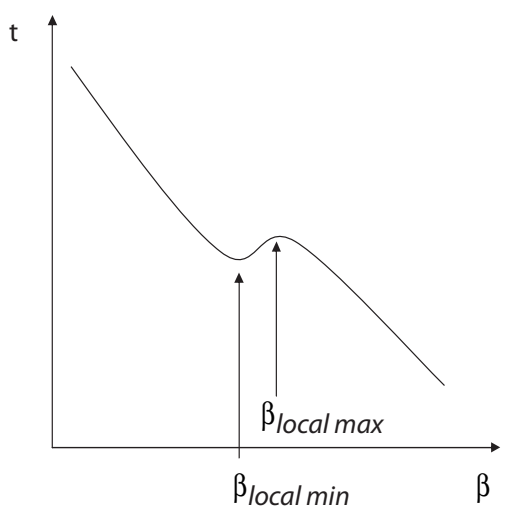

Fig. 3. Time to infect all nodes as a function of the rate $\beta$ is not a strictly decreasing function. 


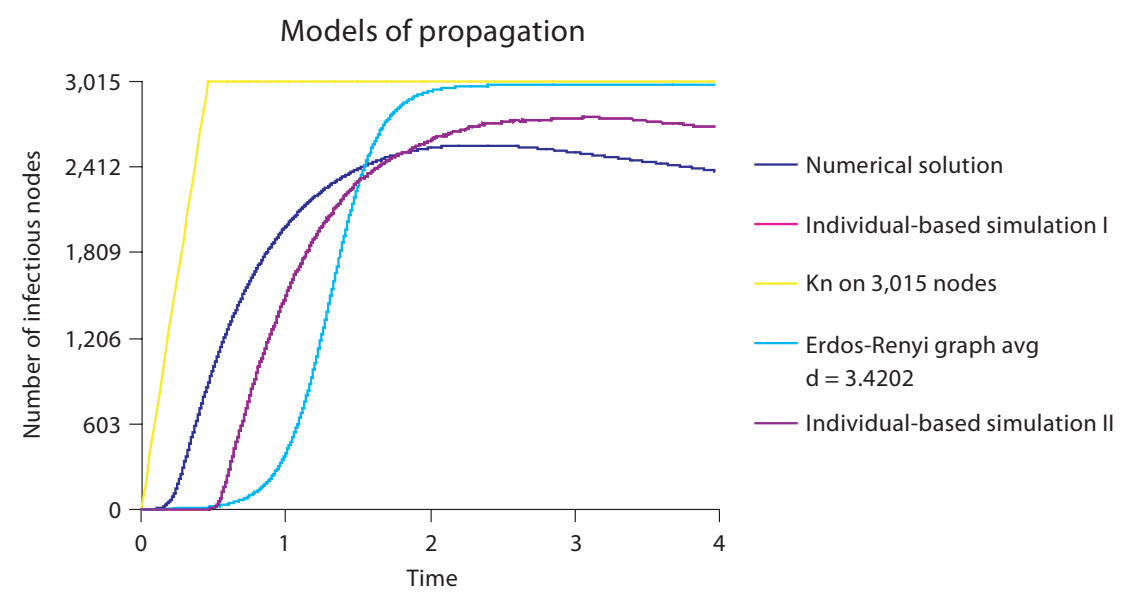

Fig. 4. SIS models of propagation - numerical solution of pair-approximation model, individual-based simulation of propagation on an Internet graph $n=3,015$ and $m=5,156$, propagation on complete graph $n=3,015$, propagation on Erdös-Renyi random graphs with $\bar{d}=3.4202$; parameters of propagation $\beta=1.8, \gamma=0.05$.

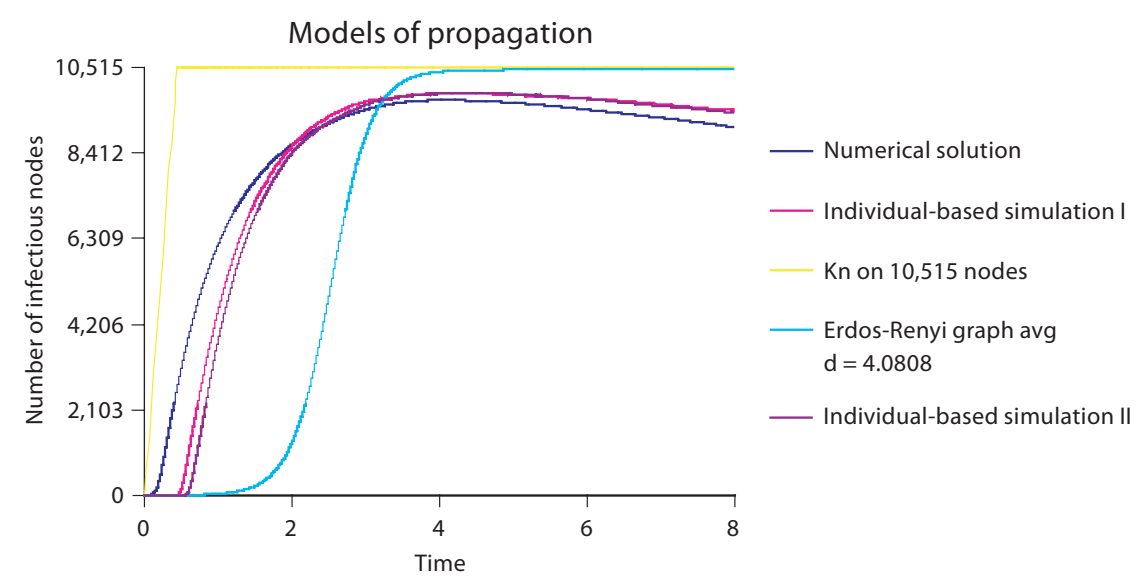

Fig. 5. SIS models of propagation - numerical solution of pair-approximation model, individual-based simulation of propagation on an Internet graph $n=10,515$ and $m=21,455$, propagation on complete graph $n=10,515$, propagation on Erdös-Renyi random graphs with $\bar{d}=4.0808$; parameters of propagation $\beta=0.9, \gamma=0.02$.

the propagation that starts from a node of minimum degree.

2) The time to propagate to all nodes increases with the increase of the order of the graph. Given two graphs $G_{1}$ and $G_{2}$, $\left|V\left(G_{1}\right)\right|<\left|V\left(G_{2}\right)\right|$, whose degree distributions follow the same scale-free distribution, have diameters $D\left(G_{1}\right)$ and $D\left(G_{2}\right)$, respectively, such that $D\left(G_{1}\right)<D\left(G_{2}\right)$. Therefore, on a graph with greater diameter the worm takes a longer time to infect all nodes.

3) The time to propagate to all nodes does not strictly decrease with the increase of the infectious rate $\beta$. In other words, there is a value of the infectious rate $\beta$ at which the function $t(\beta)$ has a local minimum, as shown in figure 3 . According to the simulation results shown in figure 2, the value of $\beta=1.5$ seems to be invariant and depends only on the exponent of the scale-free degree distribution of the graph $G$. The reason for such behaviour is that, at the local minimum, rapidly building correlations between the states of adjacent nodes hinder the propagation by lowering the number of available susceptible nodes. This observation is of particular interest as it provides the means to 'control' the propagation of a fast-spreading worm by reducing its rate to the threshold value.

Figures 4 and 5 show the number of infectious nodes as a function of time, comparing the three deterministic models with two results of the stochastic individual-based simulation. Neither the mean-field model nor the Erdös-Renyi (or a $\bar{d}$-regular graph on $n$ nodes) satisfactorily predicts the level of propagation (i.e., the number of infectious nodes at a given time). The second-order RungeKutta numerical solution of the proposed pair-approximation model (18) and (19) with results of the second preprocessing task as input matches the results of the individual-based simulation.

The model on $\bar{d}$-regular graphs underestimates the equilibrium level because it does not include the nodes of high degrees (i.e., the core of the scale-free graph). The mean-field model consistently overestimates the number of infectious nodes because the correlations and graph structure, that may inhibit propagation, are ignored. In contrast, our pair-approximation model includes both nodes of various degrees and correlations between states of nodes, and gives an accurate representation of the stochastic propagation process.

Remark: Two numerical algorithms for solving the system of differential equations - Euler's method and Runge-Kutta method - were compared. For small values of $d t$, such as 0.001 used in the simulation, even the Euler method produces good results. 


\section{Conclusion}

Developing an accurate model for the worm propagation is of critical importance not only for understanding better the worm's behaviour but also for devising techniques to contain such cyber-attacks. The existing studies include either simulation of worm propagation on a macroscopic level or a system of differential equation for propagation on ErdösRenyi and regular graphs. Moreover, in all existing models of worm propagation, it is not evident how the network structure might affect the dynamics of the stochastic propagation process. Our contribution here is 2-fold: (1) a model of propagation on a scale-free graph $G$ which takes as input: the number of nodes, number of edges, number of edges incident on nodes of certain degrees, and transitivity of the graph, and (2) implementation of an individual-based simulation for worm propagation that can be cast in different epidemiological frameworks. The accuracy of the model is tested by comparing the numerical solution (by second-order Runge-Kutta method) of the pair-approximation model to the results from the individual-based simulation on scale-free Macroscopic Internet graphs. Our model has the potential to be used in developing realistic techniques for propagation control, which is the topic of ongoing research.

\section{Acknowledgement}

Zoran Nikoloski is supported by tha European Commission-Fet Open Project DELIS IST-001907 and by the Department of Applied Mathematics, Faculty of Mathematics and Physis, Charles University, Prague, grant VZ 202-03/2060982.

\section{References}

Bauch CT: A versatile ODE approximation to a network model for the spread of sexually transmitted diseases. J Math Biol 2002; 45: 375-395.

2 Boguna M, Pastor-Satorras R, Vespignani A: Epidemic spreading in complex networks with degree correlations; in Pastor-Satorras R (ed): Lecture Notes in Physics. Berlin, Springer, 2003, vol 625, pp 127-147.

3 Chen Z, Gao L, Kwiat K: Modeling the spread of active worms; in Proc IEE INFOCOM, San Francisco, 2003.

4 CERT Coordination Center, Advisory CA-2001-26; available at: http://www.cert.org/advisories/ CA-2001-26.html 2001.

5 CERT Coordination Center, Advisory CA-2001-19; available at: http://www.cert.org/advisories/ CA-2001-19.html 2002.

6 Computer Security Institute, Ninth Annual Computer Crime and Security Survey; available at: http:// i.cmpnet.com/gocsi/db area/pdfs/fbi/FBI2004.pdf 2004

7 Cowie J, Ogielski AT, Premore BJ, Yuan Y: Global routing instabilities triggered by Code Red II and Nimda; available at: http://www.renesys.com/projects/ bgp instability 2001

8 Earnes KTD, Keeling MJ: Modeling dynamic and network heterogeneities in the spread of sexually transmitted diseases. Proc Natl Acad Sci USA 2002; 99: 13330-13335.

-9 Eguiluz VM, Klemm K: Epidemic threshold in structured scale-free networks. Phys Rev Lett 2002; 89: 108701.

10 Faloutsos M, Faloutsos P, Faloutsos C: On powerlaw relationships of the Internet topology; in Proc SIGCOMM, Cannes, 1999

11 Govindan R, Tangmunarunkit H: Heuristics for Internet map discovery; in Proc IEEE INFOCOM, Tel Aviv, 2000

12 Hethcote HW: Mathematics of infectious diseases. SIAM Rev 2000; 42: 599-653.

13 Keeling MJ: The effects of local spatial structure on epidemiological invasions. Proc R Soc Lond B 1999; 266: 859-867.

14 Kephart J0, White SR: Directed-graph epidemiological models of computer viruses; in Proceedings of IEEE Symposium on Security and Privacy, Oakland, 1991, p 343.

15 Kephart J0: How topology affects population dynamics; in Langton CG (ed): Artificial Life III. Redwood City, Addison-Wesley, 1994.

16 Kim K, Radhakrishnan S, Dhall SK: Measurement and analysis of worm propagation on Internet network topology; in Proceedings of IEEE Conference on Computer Communications and Networks, Chicago, 2004.

17 Lan K, Hussain A, Dutta D: Effects of malicious traffic on the network; in Proc PAM, La Jolla, 2003.

18 Liljenstam MN, Berk, VH, Gray RS: Simulating realistic network worm traffic for worm warning system design and testing; in Proceedings of ACM Workshop on Rapid Malcode, Washington, 2003.

19 Mirkovic J, Martin J, Reiher P: A taxonomy of DDoS attacks and DDoS defense mechanisms. ACM SIGCOMM Comput Commun Rev 2004; 34: 39-53.
20 Moore D, Paxson V, Savage S, Shannon C, Staniford S, Weaver N: Inside the Slammer Worm; available at: http://www.computer.org/security/vln4/j4wea.htm 2003.

21 Newman MEJ, Strogatz SH, Watts DJ: Random graph models of social networks. Proc Natl Acad Sci USA 2002; 99: 2566-2572

22 Nikoloski Z, Deo N: The game of cops and robbers on graphs: a model for quarantining cyber attacks. Congressus Numerantium 2003; 162: 193-215.

23 Pastor-Satorras R, Vespignani A: Epidemics and immunization in scale-free networks; in Bornholdt $\mathrm{S}$, Schuster HG (ed): Handbook of Graphs and Networks: from the Genome to the Internet. Weinheim, Wiley-VCH, 2002, pp 113-132.

24 Rand DA: Correlation equations and pair approximation for spatial ecologies; in McGlade J (ed): Advanced Ecological Theory, Principles and Applications. Oxford, Blackwell Science, 1999, pp 100-142.

25 Serazzi G, Zanero S: Computer virus propagation models; in Calzarossa MC, Gelenbe E (eds): Lectures Notes in Computer Science. Berlin, Springer, 2004, vol 2965, pp 26-50.

26 Solomon A: Epidemiology and computer viruses; available at: http://vx.netlux.org/lib/static/vdat/ epepidem.htm 1990.

27 Staniford S, Paxson V, Weaver N: How to own the Internet in your spare time; in Proceedings of the USENIX Security Symposium, Monterey, 2002, pp 149-167.

28 Vivo M, Vivo G, Koeneke R, Isern G: Internet vulnerabilities related to TCP/IP and T/TCP, Internet security attacks at the basic level. Operating Syst Rev 1998; 32: 4-15.

29 Vivo M, Carrasco E, Isern G, Vivo G: A review of port scanning techniques. ACM SIGCOMM Comput Commun Rev 1999; 29 41-48.

30 Wagner A, Dubendorfer T, Plattner B, Hiestand R: Experiences with worm propagation simulations; in Proceedings of ACM Workshop on Rapid Malcode, Washington, 2003, pp 34-41.

31 Wang C, Knight JC, Elder MC: On computer viral infection and the effect of immunization; in Proceedings of the Annual Computer Security Applications Conference, Miami, 2000.

32 Wang Y, Wang C: Modelling the effects of timing parameters on virus propagation; in Proceedings of ACM Workshop on Rapid Malcode, Washington, 2003, pp 61-66.

33 Weaver N: Potential strategies for high-speed active worms: a worst case analysis; available at: http://www. cs.berkeley.edu/,nweaver/worms.pdf, 2002.

34 Weaver N, Paxson V, Staniford S, Cunningham R: A taxonomy of computer worms; in Proceedings of ACM Workshop on Rapid Malcode, Washington, 2003.

35 Weaver N, Paxson V, Staniford S, Cunningham R: Large scale malicious code: a research agenda; available at: http://www.cs.berkeley.edu/,nweaver/ large_scale malicious_code.pdf, 2003

36 Williamson MM: Throttling viruses: restricting propagation to defeat malicious mobile code; in Proceedings of Annual Computer Security Applications Conference, Nevada, 2002, p 61 
37 Williamson MM, Leveille J: An epidemiological model of virus spreading and cleanup; in Proceedings of Virus Bulletin Conference, Montreal, 2003.

38 Yegneswaran V, Barford P, Ullrich J: Internet intrusions: global characteristics and prevalence; in Proceedings of ACM SIGMETRICS International Conference on Measurement and Modeling of Computer Systems, San Diego, 2003, pp 138-147.

39 Yook S-H, Jeong H, Barabasi AL: Modeling the Internet's large-scale topology. Proc Natl Acad Sci USA 2002; 99: 13382-13386.

40 Zou CC, Gong W, Towsley D: Code Red Worm propagation modeling and analysis; in Proceedings of ACM Conference on Computer and Communications Security, Washington, 2002, pp 138-147.

41 Zou CC, Gong W, Towsley D, Gao L: Monitoring and early detection for Internet worms; in Proceedings of ACM Conference on Computer and Communication Security, Washington, D.C., 2003. 